Monatsschr Kinderheilkd

2020 168 (Suppl 3):S204-S213

https://doi.org/10.1007/s00112-020-00950-8

Online publiziert: 10 . August 2020

(c) Springer Medizin Verlag GmbH, ein Teil von Springer Nature 2020

\section{Seit Veröffentlichung des Beitrags von Czech [5] und des Buchs von Sheffer [12] wird das Wirken Hans Aspergers fast nur mehr unter dem Aspekt seiner angeblichen Kollaboration mit der Tötungsma- schinerie der Nationalsozialisten gesehen und diskutiert. Für die Zeit nach 1945 deutet Czech sogar ei- ne Mitverantwortung Aspergers am Missbrauchsgeschehen in ös- terreichischen Kinderheimen an und fordert weitere Untersuchun- gen [5]. Dies fand auch Niederschlag in der Presse [11]. All das hat mich sehr betroffen gemacht. Es ist mir unverständlich, wie ÄrztInnen zu MittäterInnen an den national- sozialistischen Grausamkeiten werden konnten. Und nun soll mein verehrter Lehrer, Hans Asperger, Handlanger dieses Systems gewesen sein? Ich begann nachzuforschen.' Unweigerlich kam es dabei zu einer neuerlichen persönlichen Auseinan- dersetzung mit meinem Bild von der Person Hans Aspergers und "seiner" Heilpädagogik.}

In der vorliegenden Arbeit wird auf Grundlage dieses Prozesses, meiner persönlichen Kenntnis Hans Aspergers sowie des Miterlebens seiner Lehr-, Vortragstätigkeit und seines klinischen

\footnotetext{
1 Über eine differenzierte Aufarbeitung dieser Periode wurde schon an anderer Stelle berichtet (5. Jour fixe des Vereins "Politische Kindermedizin“ vom 13.02.2019, http://www.polkm.org/ newsletter/newsletter_4019.pdf, bitte Sticky Note beachten). Eine weitere diesbezügliche Darstellung findet sich im vorliegenden Heft.
}

Ernst Tatzer

Hinterbrühl, Österreich

\title{
Hans Asperger und die Heilpädagogik - ein Erfahrungsbericht
}

Wirkens in den letzten 10 Jahren seines Lebens, die Heilpädagogik, wie Asperger sie vermutlich verstand, aus eigener Sicht dargestellt. Ebenso werden die Spuren Aspergers und der Heilpädagogik in der Entwicklung verwandter Fachgebiete, insbesondere der österreichischen Kinder- und Jugendpsychiatrie aufgezeigt, und zu verschiedenen Aufarbeitungen dieses Themas wird Stellung bezogen werden.

\section{Meine persönliche Geschichte}

Hans Asperger, Jahrgang 1906, war Chef der Universitätskinderklinik Wien, als ich ihn kennenlernte. Ich hatte 1970 mit dem Medizinstudium begonnen und war im 3. oder 4. Semester auf seine Mittwochvorlesung mit dem Titel „Wege zur Menschenkenntnis“ gestoßen. Die Vorlesung begann mit der Vorstellung eines Kindes von der damaligen Heilpädagogischen Station, mit dem Asperger sich unterhielt und manchmal auch kleine Tests durchführte. ${ }^{2}$ Oft endete die Vorstellung mit der Frage: „Was willst du einmal werden?" Danach entwarf Asperger, den Studierenden gegenüber, seine eigene Beschreibung des Kindes, seiner

\footnotetext{
2 Diese Form der direkten Präsentation von Kindern ist heute zumeist abgelöst durch entsprechende Vorführungen von gefilmtem Material und wird oft sehr kritisch gesehen. Sie war zu Zeiten Aspergers in vielen Fächern des Medizinunterrichts durchaus üblich, wurde aber, abhängig von den Lehrenden, sehr unterschiedlich gehandhabt. In den Vorlesungen von Aspergerhatte ich nicht den Eindruck, dass es die Kinder sehr belastete, zumindest kam es nie zu irgendwelchen offensichtlichen Beschämungen der Kinder.
}

Begabung, möglicher konstitutioneller Aspekte und seiner „Wesensart“. Ergänzt wurde dies durch anamnestische Daten und verschiedene erhobene Befunde; es mündete in einer zusammenfassenden Beurteilung mit diagnostischen und prognostischen Aspekten. Immer erörterte Asperger anhand des vorgestellten Kindes allgemeine Fragen der psychischen Entwicklung von Kindern und Jugendlichen, setzte sie zu gesellschaftlichen Phänomenen in Beziehung und vertrat dabei auch seine persönliche Meinung. Dieser Vorlesungstermin bei Professor Asperger sollte für mich zum Fixpunkt während meines gesamten Studiums werden.

Im Jahr 1976 kam ich als junger Arzt an Aspergers Klinik, bald auch an die Heilpädagogische Station. Ich erlebte Asperger damals bei den Visiten und Besprechungen, an denen er auch noch gelegentlich nach seiner Emeritierung 1977 bis zu seinem Tod 1980 teilnahm. So konnte ich ihn über nahezu 10 Jahre persönlich erleben. Manchen war sein Agieren zu „oberlehrerhaft". Mich störte es nicht. Mich interessierte seine Art, mit Kindern zu sprechen, ihre „Wesensart“ zu erkunden und sie als eigenständige Persönlichkeiten zu verstehen. Dabei ging es ihm stets darum, ein humanistisches und positives Menschenbild respektvoll $\mathrm{zu}$ entwickeln und authentisch erlebbar zu machen. Mit mir gab es viele andere, auch aus sonstigen psychosozialen Berufsfeldern, insbesondere der Pädagogik, die sich von Asperger inspirieren ließen und bereit waren, sein Gedankengut in ihre praktische Arbeit einfließen zu lassen. 


\section{Die Heilpädagogik, wie sie damals verstanden wurde ${ }^{3}$}

Methodisch lehrte Asperger das differenzierte Schauen, Erkennen und Benennen kindlicher Phänomene sowie auch die Kraft der Entwicklung und ihre potenzielle Bedeutung. Er vermittelte uns, wie man sich persönlich einem Kind gegenüber ,ins Spiel bringt“ und schließlich in einem Wechselspiel von Beobachtungen und kindlichen Äußerungen (sprachlich, motorisch, vegetativ und kognitiv) das Kind, den Jugendlichen/die Jugendliche als Person wahrnimmt, um gleichzeitig zu reflektieren, wie dies alles auf einen selbst zurückwirkt, was man dabei spürt, und was einem dazu aus dem eigenen Inneren auftaucht.

\section{》) Asperger vermittelte die Bedeutung der reflektierenden Selbstbeobachtung}

In dieser Wechselwirkung, so lehrte Asperger, würde ein tieferes Bild über das Kind entstehen, das bei den HeilpädagogInnen $\mathrm{zu}$ umfassenden Erkenntnissen der jungen PatientInnen und ihrer Nöte, aber auch ihrer Stärken und Ressourcen sowie der möglichen und notwendigen Hilfestellungen führen würde. Asperger lehrte also, nicht nur die strukturierte Untersuchung und ihre Ergebnisse zu beachten, sondern wahrzunehmen, welches Bild des Kindes in uns durch eine reflektierende Selbstbeobachtung intuitiv entsteht. Diese zutiefst menschliche Intuition zu nützen, würde dazu führen ein „kompletteres", authentisches Bild des Kindes/Jugendlichen zu erlangen.

\section{Theoretischer Hintergrund}

All dies war natürlich geprägt von einer starken Überzeugung Aspergers von der Wirksamkeit biologischer (organischer) Faktoren, die die Möglichkeiten und Grenzen der Entwicklung eines Menschen mitbestimmen. Körper und „Seele“ waren für Asperger ein untrenn-

\footnotetext{
3 Dieser Abschnitt speist sich v. a. aus persönlichen Erinnerungen an Aspergers Vorlesungen und Vorträge.
}

bares Ganzes, der Körper Ausdruck der Seele und umgekehrt. Die Beachtung der körperlichen Erscheinungen und ihr wechselhaftes Bild während des Zwiegesprächs waren für ihn ein wichtiger Zugang zum Erkennen des Kindes. Zugrunde liegende konstitutionelle Faktoren verstand ich dabei nicht so sehr als vorbestimmtes „Schicksal“, sondern als zu beachtende Risikofaktoren, denen entsprechende Ressourcen zur Bewältigung entgegenzusetzen waren. Ebenso verband Asperger konstitutionelle Faktoren mit spezifischen Stärken und Entwicklungspotenzialen des Kindes. Immer sprach er sich für die Unterstützung der inneren Entwicklungskraft der Kinder aus. Es ging ihm nicht um lange Erklärungen, warum die Entwicklung eines Kindes in eine falsche Richtung gegangen war, sondern um die konkrete Aufhebung von Entwicklungsblockaden, seien sie intrinsisch oder extrinsisch bedingt.

\section{Spuren Aspergers}

Gefragt nach Spuren, die Hans Asperger hinterlassen hat, ist aus meiner Sicht zunächst festzuhalten, dass sein Ansinnen, "die Heilpädagogik“ als eigene Wissenschaft zu etablieren, nicht gelang. Interpretiert man aber seine Idee der „Quellströme der Heilpädagogik" ${ }^{* 4}$ als Credo einer multi- und interdisziplinären $\mathrm{Zu}$ sammenarbeit, entspricht dies durchaus einer modernen Sichtweise zur Behandlung und zur Betreuung von Kindern mit besonderen Bedürfnissen. Dementsprechend finden sich Aspergers Spuren auch in den sog. Quellwissenschaften, insbesondere in der Pädiatrie, der Kinderpsychiatrie sowie der (Heil- und Sonder-)Pädagogik.

\section{Zur Pädiatrie}

Aspergers Einfluss in der Pädiatrie bestand hauptsächlich darin, viele KollegInnen in ihrer Entwicklung und ihrer

\footnotetext{
4 „„... es (sind) vielmehr 5 Wissenschaften, welche die Quellströme der heilpädagogischen Lehre bilden: zwei ärztliche Sondergebiete, die Psychiatrie und die Kinderheilkunde, dann die Psychologie, die Sozialwissenschaft und die Pädagogik" $[2$, S.1].
}

Sichtweise auf das Kind stark beeinflusst zu haben (z.B. in den Fortbildungswochen in Obergurgl). Als Klinikchef gab er den MitarbeiterInnen weitgehend freie Hand, ihre Spezialitäten zu entwickeln und voranzutreiben. Was ihm nicht gelang, war, die Heilpädagogik als integrierten Bestandteil einer modernen Pädiatrie zu etablieren. Dies wohl auch deshalb, weil sich gerade in den Jahren nach 1960 innerhalb der Pädiatrie hauptsächlich organische Subspezialitäten (Kardiologie, Pulmologie, Nephrologie etc.) ausbildeten, die in ihrer rasanten Entwicklung eine hohe Attraktivität auf junge KlinikärztInnen ausübten und die „psychischen Aspekte“ innerhalb der Pädiatrie in den Hintergrund treten ließen. Meiner Meinung nach verabsäumte es die Pädiatrie in Österreich zu dieser Zeit, eine eigenständige pädiatrische Psychosomatik zu entwickeln. Asperger hätte mit seinen Überlegungen dazu wohl Grundsätzliches beitragen können. Erst mit dem Gedanken des Kinderschutzes und der Gründung entsprechender Kinderschutzgruppen etablierte sich wieder ein gewisses Bewusstsein für psychosoziale Zusammenhänge. Heute wird vielerorts versucht, eine pädiatrische Psychosomatik (in Kooperation mit der Kinder- und Jugendpsychiatrie) als wesentliches Feld innerhalb der Pädiatrie einzurichten, was sich sowohl als hilfreich wie auch als dringend notwendig herausstellt.

\section{Zur Kinderpsychiatrie (heute Kinder- und Jugendpsychiatrie und psychotherapeutische Medizin)}

Die Anfänge der heutigen Kinder- und Jugendpsychiatrie und psychotherapeutischen Medizin (KJPP) liegen in Österreich in der Heilpädagogischen Station (Erwin Lazar, Valerie Bruck, Hans Asperger $)^{5}$ und der kinderpsychiatrischen Station der Klinik Hoff (Walter Spiel $)^{6}$. Beide waren anfänglich Teil ih-

\footnotetext{
5 Im Jahr 1911 unter Erwin Lazar (1877-1932) mit Eröffnung der Wiener Universitätskinderklinik (erster Leiter Clemens von Pirquet [1874-1929]) gegründet.

6 Im Jahr 1949 Errichtung eines Kinderzimmers, 1951 Erweiterung zu einer 12-Betten-
} 
Monatsschr Kinderheilkd 2020 - 168 (Suppl 3):S204-S213 https://doi.org/10.1007/s00112-020-00950-8

(c) Springer Medizin Verlag GmbH, ein Teil von Springer Nature 2020

\section{E. Tatzer}

\section{Hans Asperger und die Heilpädagogik - ein Erfahrungsbericht}

\section{Zusammenfassung}

Die Auseinandersetzung mit den Darstellungen von Czech und Sheffer über Hans Aspergers Rolle während des Nationalsozialismus (NS) bedingte unweigerlich eine neuerliche persönliche Beschäftigung mit dem eigenen Bild von Asperger und seinem Wirken. Über 10 Jahre habe ich - zunächst als Student, dann als Mitarbeiter seiner Klinik Asperger persönlich erlebt. Sein beispielhafter Umgang mit den Kindern, seine daraus abgeleiteten Darstellungen und Erörterungen sowie die Vermittlung des umfassenden Lehrgebäudes der Heilpädagogik und eines höchst humanen Menschenbilds als Grundlage jedes Handelns inspirierten mich und viele meiner Generation.

Auch wenn es Asperger nicht gelang, die Heilpädagogik als integrierten Bestandteil innerhalb der Pädiatrie zu verankern, lassen sich Spuren Aspergers in den Biografien vieler KinderärztInnen finden. Noch größeren Einfluss hinterließ er im Fachgebiet der Kinder- und Jugendpsychiatrie. Anhand der Entwicklung dieses Fachgebiets wird in dieser Arbeit der Beitrag der Heilpädagogischen Station als eine ihrer zwei Wurzeln aufgezeigt. Dabei wird die Heilpädagogische Station mit ihrem geregelten Tagesablauf, dem interdisziplinären Team und ihrer kindzentrierten Arbeitsweise beschrieben. Ebenso wird auf die Diskrepanz zwischen den Darstellungen Aspergers, was Heilpädagogik sei, und einer, durch rigide Auslegung teilweise von seinen Vorstellungen abweichenden Umsetzung in den 1970er-Jahren Stellung genommen. Die inspirierende Rolle Aspergers auf die Pädagogik wird anhand der Entwicklung und Tätigkeit der Heilpädagogischen Gesellschaft Österreich dargestellt.
Nicht zuletzt werden Kritikpunkte zu Asperger und der Heilpädagogik hinterfragt. Dabei wird v.a. die ausschließlich zeitgeschichtliche Perspektive aus Akten, Krankengeschichten und Literatur in Bezug auf Aspergers Wirken ohne ausreichende Diskussion der damaligen Umstände problematisiert, die meiner Ansicht nach Anlass für Fehlinterpretation geben kann. Gleiches gilt für den Verzicht auf das Heranziehen von ZeitzeugInnen. Weitere Aufarbeitungen, v.a. die Heilpädagogik betreffend, sollten in Zusammenarbeit von ExpertInnen der Zeitgeschichte mit jenen der Medizin (Heilpädagogik) erfolgen.

Schlüsselwörter

Nationalsozialismus · Lehrtätigkeit · Pädiatrie · Kinder- und Jugendpsychiatrie. Geschichte

\section{Hans Asperger and therapeutic pedagogy—A report on personal experiences}

\section{Abstract}

The discussion about the publications by Herwig Czech and Edith Sheffer about Hans Asperger's role during the National Socialism regime resulted in a renewed personal occupation with my own picture of Hans Asperger and his work. Over a period of 10 years, I personally experienced Asperger, first as a student then as an assistant at his clinic. Asperger's exemplary handling of children, his special way of presentation and explanation to the students deeply impressed me. The way he developed the comprehensive field of therapeutic pedagogy and his profoundly humane image of man as the foundation of all actions also inspired me as well as many others of his generation.

Asperger's influence can be found in the biographies of many pediatricians even if he did not succeed in anchoring therapeutic pedagogy as an integrated part of pediatrics; however, he had a greater influence in the field of child and adolescent psychiatry. The development of this field elucidates the contribution of therapeutic pedagogy as one of its two roots. In this article, the therapeutic pedagogy ward at the Children's University Clinic of Vienna with its structured daily routine, the interdisciplinary team and its childcentered way of treatment are described. The discrepancy between Asperger's presentations as to what constitutes therapeutic pedagogy and the partly deviating reality in the 1970 s is discussed. Asperger's inspiring influence on pedagogy in Austria and the development and activities of the Austrian Society for Therapeutic Pedagogy are described.
Moreover, various points of criticism about Asperger and therapeutic pedagogy are questioned. Especially, I find fault with the purely contemporary perspective in the interpretation of files, medical records and literature regarding Asperger's work, which may have led to misinterpretations. In addition, no contemporary witnesses were consulted for these publications. Further studies concerning therapeutic pedagogy should be carried out in a collaboration between historians and experts from the medical field (therapeutic pedagogy).

Keywords National Socialism · Teaching · Pediatrics . Child and adolescent psychiatry $\cdot$ History 
res Mutterfachs Kinderheilkunde bzw. Psychiatrie/Neurologie. Im Jahr 1975 wurde die Kinderneuropsychiatrie als neue Subspezialität mit 3-jähriger $\mathrm{Zu}$ satzausbildung $\mathrm{zu}$ den Mutterfächern in das Ärztegesetz aufgenommen. ${ }^{7}$ Im gleichen Jahr erfolgte die Gründung der Universitätsklinik für Kinder- und Jugendneuropsychiatrie, mit Walter Spiel (1920-2003) als Leiter, und des interdisziplinären Instituts für Heil- und Sonderpädagogik. Dieses Institut war Aspergers letzter Versuch, Heilpädagogik als interdisziplinäres Fachgebiet $\mathrm{zu}$ etablieren. ${ }^{8}$ Im Jahr 2006 erfolgte nach langem Ringen - es waren gerade die PädiaterInnen, die sich dagegenstellten, weil sie „ihre“ Heilpädagogik nicht hergeben wollten - die Anerkennung zum Sonderfach Kinder- und Jugendpsychiatrie und 2018 die Erweiterung zur Kinder- und Jugendpsychiatrie und psychotherapeutischen Medizin. ${ }^{9}$ Zwischen den beiden Schulen "Asperger“ und „Spiel“ herrschten ein Konkurrenzkampf und ein starkes gegenseitiges Misstrauen. Obwohl im selben Gebäude des neuen Klinikums untergebracht (die Heilpädagogische Station im Parterre, die Neuropsychiatrie im ersten und zweiten Stock), gab es lange Zeit wenig Kontakt und noch weniger Austausch miteinander. Die von „oben“ meinten, die anderen würden nur „graue“ Pädagogik betreiben; die von „unten“ sagten, die „oben“ würden die Kinder an der Station nicht kindgerecht und adäquat betreuen. Zwar kam es 1978 zum ersten und daraufhin

Station, 1972 Extraordinariat und Abteilung für Kinderneuropsychiatrie.

7 In der Anlage 8 ist unter Z.8 Kinderheilkunde in der Spalte Anmerkungen folgende Anmerkung einzufügen: „Jene Fachärzte für Kinderheilkunde, die den Zusatz ,(Kinderneuropsychiatrie)' führen wollen, haben zusätzlich eine einjährige Ausbildung in Psychiatrie und eine zweijährige Ausbildung an einer Abteilung für Neuropsychiatrie des Kindesalters nachzuweisen." https://www.ris.bka.gv.at/ Dokumente/BgblPdf/1975_529_0/1975_529_ 0.pdf.Zugegriffen:23.04.2020.

8 Dieses Institut hatte nur eine kurze Lebensdauer und ging nach etwa 20 Jahren im Institut für Erziehungswissenschaften auf.

9 https://www.ris.bka.gv.at/GeltendeFassung. wxe?Abfrage $=$ Bundesnormen\&Gesetzes nummer=20009186.Zugegriffen:23.04.2020. zu jährlichen Treffen, die dem Austausch dienen sollten, später zur Gründung einer Arbeitsgemeinschaft (AG) Kinderund Jugendneuropsychiatrie und in der Folge zu einer gleichnamigen wissenschaftlichen Gesellschaft. Aber wirklich verbunden hat die beiden Lager erst der gemeinsame Kampf um die Anerkennung eines eigenen Sonderfachs, der bereits von der nachfolgenden Generation betrieben wurde.

\section{Zur Heilpädagogischen Station}

Der Vorwurf einer „grauen“ Pädagogik ist im Nachhinein in manchen Aspekten für mich nachvollziehbar, kam ich doch 1976 an eine Station, die für mich mit einer zeitgemäßen Heilpädagogik nicht im Einklang stand. Die Beibehaltung der alten Regeln, dass die Kinder während des stationären Aufenthaltes ihre Eltern nicht sehen und nur über Briefwechsel mit ihnen in Kontakt treten konnten, das Tragen einheitlicher Stationskleidung der Kinder, die immer versperrte Stationstür und das rigide Regime des stationsführenden Oberarztes, das eigenen Initiativen keinen Raum ließ, vergällte etlichen KollegInnen die Arbeit an der Station.

Aber es war auch viel Positives vorhanden: Der Alltag der Station war geprägt von einer kindgemäßen und überschaubaren Tagesstruktur, die einen regelmäßigen Kindergarten- und Schulbesuch, Ruheperioden, Aufgabensituationen, aber auch etliches an Freizeit- und Festivitäten umfasste. Viel Wert und Aufmerksamkeit wurde auf das Beheben schulischer Rückstände und Defizite gelegt, in der Freizeit auf Bewegung und Spiele, auch im Freien, sowie auf kreative Medien, wie Zeichnen, Malen, Basteln, außerdem auf das Lesen und Hören spannender Geschichten.

Aufgenommen wurden Kinder vom Kindergartenalter bis zum Ende der Schulpflicht mit praktisch allen Verhaltens-, Entwicklungs- und psychosomatischen Störungen, auch mit kinderpsychiatrischen Krankheitsbildern im engeren Sinn. Da sich bei älteren Kindern die Auffälligkeiten oft im Kontext der Schule zeigten, stand der gegenseitige Austausch mit der Herkunftsschule auf der Tagesordnung, wenn nötig auch der mit dem Jugendamt.
Begleitet wurde der meist 4- bis 6-wöchige Aufenthalt von heilpädagogischen Gesprächen der ÄrztInnen und PsychologInnen mit den Kindern. Die PsychologInnen führten auch die entsprechenden Tests durch. Einzeltherapeutische Ansätze waren verhaltens- und/oder suggestivtherapeutisch geprägt. Gezielte Psychotherapieangebote für Kinder und ihre Familien, wie z.B. systemische (familientherapeutische) oder klientenzentrierte Therapieformen waren an der Station damals noch nicht etabliert. Ergänzt wurde das Angebot durch Logopädie, später auch Ergo- und Physiotherapie. Nach etwa 3 Wochen wurde jedes Kind im Team (gelegentlich unter dem Vorsitz Aspergers) ausführlich besprochen, wobei versucht wurde, aus den Einzelmeinungen ein Gesamtbild zu finden und entsprechende Vorschläge für das „Danach“ zu erarbeiten.

\section{》) Im kindgerechten Klima der Heilpädagogischen Station fühlten sich die Patienten wohl}

Im Allgemeinen hatte man den Eindruck, dass ein kindgerechtes Klima herrschte und die Kinder sich wohlfühlten. Manche Auffälligkeiten fielen wie von selbst $\mathrm{ab}$, wurden doch die Kinder von Anfang an von der strukturierten Gruppenatmosphäre mitgetragen. Kinder mit Mutismus fingen wie von selbst wieder zu sprechen an; Kinder, die vorher die Schule verweigert hatten, besuchten vom ersten Tag an die Stationsschule, als hätten sie nie ein Problem gehabt; ängstliche Kinder produzierten in kürzester Zeit in der täglichen Turnstunde Übungen, die ihnen zuvor keiner zugetraut hätte. Sehr bald musste ich allerdings feststellen, dass die Transferierung des Erreichten in das ursprüngliche Umfeld, v. a. bei älteren Kindern, oft nur schwer gelang. Externe Schulwechsel brachten nur bedingte Erfolge, und viele Eltern waren zu wenig darauf vorbereitet, was zu tun sei, wenn erneut Schwierigkeiten auftreten würden. Auch traute man den Eltern wenig zu und suchte immer wieder das Heil in der sog. Administration der Kinder in Heimen oder Internaten, in der Erwartung, dass die positiven Er- 
folge sich dort weiterstabilisieren mögen. Die Unterbringung in solchen Institutionen geschah fast ausschließlich mit $\mathrm{Zu}$ stimmung der Eltern. Auch handelte es sich nicht um "geschlossene Heime“. Die nunmehr offensichtlichen verheerenden Zustände von Missbrauch und Demütigungen von Kindern in vielen Heimen waren damals noch nicht bekannt. Zwar verfasste Leirer 1976 im Auftrag der Magistratsabteilung 11 nach 2-jährigen Untersuchungen von 34 Heimen im Bereich der Stadt Wien einen Bericht, der nichts an Deutlichkeit vermissen ließ [9]. Doch wurden daraus von den Verantwortlichen keine Konsequenzen gezogen, und der Bericht durfte nur zensuriert veröffentlicht werden. ${ }^{10}$

Die Auseinandersetzungen mit den Kindern konnten teilweise vonseiten des an sich sehr zugewandten Pflegepersonals ziemlich lautstark werden. Ich habe aber dabei auf der Heilpädagogischen Station nie eine körperliche Misshandlung von Kindern wahrgenommen. Manchmal endeten solche Episoden allerdings mit einer Beschämung für das Kind, was aus meiner Sicht niemals Teil der Heilpädagogik war, wie ich sie von Asperger gehört und verstanden habe.

\section{》) Asperger schien die \\ Einengungen "seiner \\ Heilpädagogik" nicht wahrgenommen zu haben}

Allerdings hat Asperger die rigiden Begrenzungen seitens der Stationsleitung, die eine Weiterentwicklung der Heilpädagogik zu dieser Zeit stark behinderten oder unmöglich machten, geduldet oder übersehen. Er ließ es zu, dass auf sein humanistisches Menschenbild in der praktischen Arbeit damals z.T. nicht entsprechend ausreichend eingegangen wurde, und hielt an dem rigiden und jeglicher Öffnung entgegenstehenden, stationsführenden Oberarzt fest. Warum dies so war, kann ich nicht beantworten.

\footnotetext{
10 Interview mit Irmtraut Karlsson Ö 1, Morgenjournal,19.10.2011 https://oe1. orf.at/artikel/288741/Studie-Heime-alsKindergefaengnisse.Zugegriffen:23.04.2020.
}

Dies ist wahrscheinlich der schwärzeste Punkt in meinem Bild von Hans Asperger.

Denn für mich brachte gerade die Emeritierung von Hans Asperger einen Wendepunkt. Der stationsführende Oberarzt wurde auf Weisung des neuen Klinikchefs abgesetzt; ihm folgte der habilitierte Kinderarzt und Neuropädiater Christoph Groh $\left({ }^{\star} 1924\right)$ als neuer Stationsleiter. Dieser war an einer Öffnung und Weiterentwicklung der Station höchst interessiert und förderte neue, vielversprechende Initiativen von jungen MitarbeiterInnen, denen er ein väterlicher Mentor war. So konnten die Systemische Familientherapie und andere Psychotherapieformen an der Station eingeführt und angewandt werden. Sie standen gleichberechtigt neben dem heilpädagogischen Handeln und ergänzten es. Die Station wurde für Besuche der Eltern und Ausgänge mit Übernachtung zum Wochenende geöffnet, die Stationskleidung abgeschafft. Die Zusammenarbeit mit der gesamten Kinderklinik wurde verstärkt, sodass zunehmend auch schwere psychosomatische Krankheiten in Kooperation mit SpezialistInnen der Pädiatrie behandelt werden konnten. Dass die Modernisierung unter Groh relativ rasch gelang, ist wohl ein Zeichen dafür, dass an der Station viele Menschen tätig waren, die den Geist seiner Heilpädagogik verinnerlicht hatten. Dass Asperger diese Veränderungen, als Emeritus an der Station immer wieder anwesend, keineswegs abgelehnt hat, spricht wohl dafür, dass er die vorher bestehende Einengungen „seiner Heilpädagogik“ nicht wahrgenommen hatte.

Als Groh selbst zum supplierenden Leiter der Kinderklinik bestellt wurde, holte er Werner Leixnering $\left({ }^{*} 1950\right)$, damals Oberarzt an der Klinik Spiel, als Stationsleiter an die Heilpädagogische Station, die bald „Heilpädagogik und Psychosomatik“ heißen sollte. Leixnering entwickelte sie weiter, $\mathrm{zu}$ einer modernen psychosomatisch orientierten Abteilung, und zwar durchaus aufheilpädagogischer Basis, indem er Grundsätze der Heilpädagogik beibehielt und um moderne diagnostische und therapeutische Ansätze erweiterte. Dass die Station später unter einem neuen Klinikchef nur noch den Status einer Tagesklinik innehatte und damit an Bedeutung und Versorgungskraft verlor, ist eine andere Geschichte.

Beim Aufbau der eigenen kinderpsychiatrischen Abteilung in der Hinterbrühl (Niederösterreich) war der heilpädagogische Weg, der auch von meinem Vorgänger, Erwin Schmuttermeier (1931-2013), gepflegt worden war, eine wichtige Grundlage.

Der finale Schritt zu einer kinderund jugendpsychiatrischen Abteilung nach gemeinsamer Konzeptionierung durch meinen Nachfolger, Rainer Fliedl $\left({ }^{*} 1957\right)$, vollzogen. Zuvor war die Genehmigung der Kinder- und Jugendpsychiatrie als Sonderfach erteilt worden und die formale Angliederung der Heilpädagogik als Abteilung für Kinder- und Jugendpsychiatrie und -psychotherapie an das Landeskrankenhaus Mödling erfolgt.

Kinder- und Jugendpsychiatrien i.Allg., die ihre Räumlichkeiten als Wohngruppen gestalten, die Wert auf einen strukturierten Tagesablauf legen und die Pflegekräften, PädagogInnen und anderen Berufsgruppen gleichberechtigtes Agieren ermöglichen, sind erkennbare Anzeichen, die Hans Asperger in der österreichischen Kinderund Jugendpsychiatrie hinterlassen hat. Tatsächlich wird so das von Asperger angestrebte interdisziplinäre Arbeitsklima verwirklicht.

\section{Zur Pädagogik}

Mehr noch als in der Pädiatrie identifizierten sich viele Personen aus Bereichen der Pädagogik, also LehrerInnen, SonderschullehrerInnen, SozialpädagogInnen und KleinkindpädagogInnen mit Aspergers Weg der Heilpädagogik. Das Forum dazu bildete die „Arbeitsgemeinschaft für Heilpädagogik“, deren Präsident Asperger durchgehend bis zu seinem Tod 1980 war. Sie wurde in Anlehnung an die von Theodor Heller (1869-1938) ins Leben gerufene „Österreichische Gesellschaft für Heilpädagogik“ im Jahr 1948 als solche neu 
gegründet. ${ }^{11}$ Im Jahr 1980 erfolgte eine Umstrukturierung in eine Dachorganisation mit selbstständigen Landesgruppen, um den föderalistischen Aspekt besser berücksichtigen zu können. Die heutige Bezeichnung „Heilpädagogische Gesellschaft Österreich“ (HPGÖ) ${ }^{12}$ wurde 1994 gewählt. Seit 1975 veranstaltet die Gesellschaft jedes zweite Jahr (zuletzt 2018 in Vöcklabruck) den Internationalen Heilpädagogischen Kongress. Sie veröffentlicht vierteljährlich die Zeitschrift Heilpädagogik.

Im Unterschied zur deutschen und zur Schweizer Heilpädagogik, die die Heilpädagogik ausschließlich als Teil der Pädagogik betrachteten, verstand sich die österreichische Heilpädagogik im erweiterten Sinne Aspergers, nämlich auf den fünf Quellströmen beruhend. Allerdings kam der Hauptanteil der Mitglieder aus dem Sonderschulbereich, sodass das behinderte Kind und seine Familie zunehmend in den Fokus rückten. Interdisziplinarität ergab sich hinsichtlich der Versorgung behinderter Kinder durch die Zusammenarbeit mit darauf spezialisierten ÄrztInnen, PsychologInnen, funktionellen TherapeutInnen, SozialpädagogInnen und SozialarbeiterInnen. Aspergers Schwerpunkt der Verhaltensauffälligkeiten trat allerdings immer mehr in den Hintergrund der Arbeit, abgesehen von Personen, die an Heilpädagogischen Stationen tätig waren. Zuletzt hat aber auch dieser Schwerpunkt wieder mehr an Bedeutung gewonnen.

Von vornherein berief sich die HPGÖ auf folgende 3 Ziele: Wissenserweiterung (Forschung), Fortbildung der Mitglieder sowie Vertretung der Anliegen von Kindern und Jugendlichen mit Behinderungen samt ihren Familien. Aufgrund der nur kurzen Zusatzausbildung von SonderschullehrerInnen (selbst diese war für den Unterricht in der Sonderschule nicht verpflichtend), war deren Fortbildung eine der wesentlichen Aufgaben der Gesellschaft, ebenso die Forderung nach Ausbau des Sonderschulwesens, v.a. in

\footnotetext{
11 http://www.heilpaedagogik.at/entstehung. php.Zugegriffen:07.04.2020.

12 Ich war von 2008 bis 2014 Präsident und bin seit Mai 2016 Ehrenpräsident dieser Gesellschaft.
}

den Bundesländern. Damit ergab sich auch eine starke Verflechtung der Funktionäre der Gesellschaft mit Repräsentanten der entsprechenden Schulbehörden. Fragen der Interdisziplinarität hatten einen besonderen Stellenwert. Aspergers Rolle war auch hier die eines Inspirators. Mitunter wurde seine Lehre allerdings im Sinne einer Überbetonung der Bedeutung der medizinischen Grundlagen missverstanden, ${ }^{13}$ eine Tendenz, der Asperger [3] in seinem letzten Vortrag auf dem 3. Heilpädagogischen Kongress deutlich widersprach. Dort wies er neuerlich auf die Zusammenarbeit der „Quellströme“ der Heilpädagogik als gleichberechtigte Berufsfelder „auf Augenhöhe“ hin und entwarf ein optimistisches Bild der Entwicklung neuer Methoden auf jedem Gebiet, insbesondere auf dem der Pädagogik. Darüber hinaus sprach er sich für die enge Zusammenarbeit mit der Familie aus, speziell mit der Mutter, der er die eigentliche Expertise für das Kind zuschrieb. Jedenfalls sollte man auch alles daransetzen, die Wünsche und Bedürfnisses des Kindes, gerade des behinderten, zu erkennen und in die Behandlung einzubeziehen.

\section{》) Hans Asperger galt \\ als Vertreter eines zutiefst humanistischen (christlichen) Menschenbilds}

Mit der Integrationsbewegung kam es zu einer inneren Krise der Gesellschaft. Die Affäre Wurst war zusätzlich eine große Belastung. ${ }^{14}$ Heute versteht sich die Gesellschaft als interdisziplinäre Vereinigung, deren Tätigkeitsschwerpunkt in den Bundesländern liegt. Als eines ihrer Hauptziele tritt sie für die Inklusion behinderter Menschen ein.

Als Mitbegründer der „Internationalen Pädagogischen Werktage“ in Salzburg 1950 unterstützte Asperger ein zusätzliches Forum, in dem es um Reflexion und Weiterentwicklung der Pädagogik

\footnotetext{
13 So manch eine/einer dieser PädagogInnen verstand sich als "kleiner Mediziner".

${ }^{14}$ http://www.heilpaedagogik.at/archiv_ obmann.php.Zugegriffen:07.04.2020.
}

im interdisziplinären Rahmen geht. Die „Werktage“ werden jährlich von mehr als 1000 Personen aus sämtlichen Gebieten der psychosozialen Versorgung besucht. Auch hier galt Hans Asperger als Vertreter eines zutiefst humanistischen (christlichen) Menschenbildes.

\section{Zur Kritik an Aspergers Sichtweisen}

Bei der Kritik an Aspergers Sichtweisen werden gern Zitate aus seinem Lehrbuch Heilpädagogik herangezogen. Es erschien erstmals 1952 und in 4 weiteren Ausgaben: 1956 (ergänzt und erweitert), 1961 (überarbeitet und erweitert), 1965 und 1968 (mit Bedauern Aspergers unverändert). Als Grundlage der folgenden Diskussion verwende ich die letzte Ausgabe [2] und ergänze den Diskurs durch persönliche Bemerkungen und Erfahrungen.

Das Buch Heilpädagogik von Hans Asperger ist für heutige Verhältnisse schwierig zu lesen. Es ist in einem Erzählstil gehalten, ohne Abbildungen, sehr raumsparend gegliedert und unter Verwendung nicht mehr gebräuchlicher Ausdrücke. Im allgemeinen Teil präsentiert sich Asperger als genauer Beobachter von Kindern, die er in bildhafter Sprache beschreibt. Dabei verwendet er gelegentlich Bilder, die wir heute nicht mehr gebrauchen würden. Wer die Ausdauer hat, die Beschreibungen sorgfältig $\mathrm{zu}$ lesen, erkennt das Bemühen Aspergers, das jeweilige Kind aus mehreren Perspektiven zu schildern, und gewinnt schlussendlich ein sehr plastisches Bild seiner Zustände und Symptome. Es handelt sich um eine Beschreibungskunst, die heute weitgehend verloren gegangen ist und die durch, wie Asperger sagen würde, „seelenlose“ Diagnoseschemata ersetzt wurde. Ähnlich agiert Asperger im speziellen Teil des Buches unter Heranziehung des ihm zur Verfügung stehenden medizinischen Wissens seiner Zeit (letzte redigierte Ausgabe 1961). Im Großen und Ganzen gilt die von ihm gewählte Systematik auch heute noch, wobei natürlich Ausdrücke, wie „Psychopath“, „Neuropath“ u. A. aus dem Vokabular verschwunden sind. 
Freilich finden sich auch Irrtümer bzw. Ausdrücke, die zu Missverständnissen führen können. Ein großer Teil der Diskussion um die Bewertung von Aspergers Lehrmeinungen nimmt sein Verständnis von ,angeboren“, „Konstitution“ und „Determinismus“ ins Visier. Letztendlich geht es um die der Geschichte der Psychiatrie immanente Frage nach dem Einfluss von Vererbung und Milieu, heute eher Ausstattung und Umwelt genannt. Im Kapitel „Allgemeine Ursachenlehre“ nennt dies Asperger den „Streit darum, was das Schicksal der einzelnen Persönlichkeit in stärkerem Maße bestimmt: die inneren, in der Konstitution verankerten, anlagemäßig gegebenen Faktoren oder das Milieu, also all das, was vom Augenblick der Zeugung an von außen her auf den Menschen einwirkt" [2, S. 42]. In der nachfolgenden Diskussion würdigt Asperger beide Aspekte, weist allerdings eher auf die konstitutionellen, organischen Faktoren hin. Die Auflösung der Dichotomie erfolgt in Aspergers typischer Art nicht als wissenschaftliche Erklärung, sondern als Plädoyer für ein humanistisches Menschenbild: „Wir fühlen uns gedrängt, auch an dieser Stelle ein Bekenntnis zur Freiheit der menschlichen Persönlichkeit abzulegen, welche Freiheit diese Geschlossenheit von äußeren und inneren Gegebenheiten aufzuheben imstande ist und durch welche der Mensch, sosehr er auch an Erbe und Umwelt gebunden ist, sich doch, und sei es nur in wenigen entscheidenden Augenblicken, von beidem distanzieren mag“" [2, S. 51].

In der Literatur [5, 7] werden Gutachten Aspergers zitiert, aus denen sich eine einseitige Betonung der vererbten (konstitutionellen) Faktoren ableiten lässt. Inwieweit es sich um Einzelfälle handelt oder eine solche Tendenz tatsächlich festzustellen ist, wäre Thema für eine eigene Forschungsarbeit, die in Kooperation von HistorikerInnen mit MedizinerInnen geleistet werden könnte. In der Frage der konstitutionellen Faktoren wird hervorgehoben [5, 7], wie Asperger das sexualisierte, „verführende“ Verhalten von Mädchen und jungen Frauen, die sexuell missbraucht wurden, ihrer angeborenen Konstitution zuschreibt. Heute wissen wir, dass dieses Verhalten oft mehr Folge des meist mehrfachen sexuellen Missbrauches ist als dessen Ursache [10, S. 30]. Die entsprechenden Studien dazu haben allerdings erst nach dem Tod Aspergers begonnen, und er selbst hat, wie viele andere auch, die diesbezüglich damals gängige Lehrmeinung nicht infrage gestellt. So schreibt Bange [4] zusammenfassend: „Die Erforschung von sexualisierter Gewalt in Deutschland ist durch

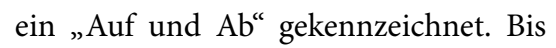
in die jüngste Vergangenheit hinein haben Wissenschaftlerinnen und Wissenschaftler meist bestehende gesellschaftliche Vorurteile verstärkt und die betroffenen Mädchen und Frauen und Jungen und Männer abgewertet. Erst als durch die Selbsthilfebewegung und die Frauenbewegung sexualisierte Gewalt in den 70iger und 80iger Jahren zu einem öffentlichen Thema gemacht wurde, änderte sich die Situation." Wir wissen heute auch, wie sehr sich traumatisierende Erlebnisse im Gehirn einprägen und so das Bild einer nahezu unveränderbaren Persönlichkeitsstörung produzieren. ${ }^{15} \mathrm{Hier}$ irrte Asperger also, wie viele seiner ZeitgenossInnen auch. Dies macht deutlich, wie notwendig eine feministisch geprägte Aufarbeitung vieler noch heute geltender Vorurteile in Medizin und Geschichte ist.

Aspergers Empfehlungen zu „strikter Führung" und zu Fremdunterbringung (oft verwechselt mit geschlossener Führung, also geschlossenen Anstalten), gaben Czech [5] Anlass zu der Kritik, Asperger hätte zu einer Fülle nichtnotwendiger Heimunterbringungen beigetragen. Damit wird eine indirekte Mitverantwortung Aspergers für psychischen, physischen und sexuellen Missbrauch in Heimen in den Raum gestellt. In Wahrnehmung des humanistischen Menschenbilds Aspergers und seines Auftretens dafür in zahlreichen Publikationen und Vorträgen kann ich diese Meinung in keiner Weise nachvollziehen.

Möglicherweise könnten Aspergers Wortwahl und verkürzte Darstellungen

\footnotetext{
15 https://www.degpt.de/informationen/fuerbetroffene/trauma-und-traumafolgen/wie$\%$ C3\%A4u\%C3\%9Fern-sich-traumafolgest $\%$ C3\%B6rungen/posttraumatische-belastungs st\%C3\%B6rung/.Zugegriffen:07.04.2020.
}

zu dem Missverständnis verleiten, dass er eine rigide Disziplinierung mit dem Ziel einer gesellschaftlichen Normalisierung (Anpassung) und optimalen Leistungserbringung befürwortet hätte. So schreibt Asperger 1940,,im Gegenteil, eine solche Einstellung führt $\mathrm{zu}$ einer höchst aktiven Pädagogik, die in straffer Führung alles aus dem abartigen Kind herausholt, was nur in ihm steckt, die in angepaßter Steigerung seine Kräfte übt und stärkt..“, „... Dazu gehört eine Führung, die so lückenlos ist, daß es keine Gelegenheit zu Unfug und dissozialen Streichen; dazu eine Führung, die dem Gefährdeten so lange die Verantwortung abnimmt, bis der eigene Halt, die eigene Verantwortung nachgereift sind (das ist freilich oft nur in einer Anstalt möglich)“ [1, S. 355].

\section{》) Asperger sprach sich für "eine menschliche Ordnung [ohne] starren Drill und Zwang" in Heimen aus}

In seinem Lehrbuch führt Asperger zur „Problematik heilpädagogischer Anstalten für kriminelle Kinder und Jugendliche" seine Haltung zur Heimerziehung klarer aus. Dort spricht er sich für „eine menschliche Ordnung [ohne] starren Drill und Zwang“ aus, denn dieser würde „erst recht gefährliche Ressentiments erzeugen, schwere Aggressionen auslösen, dissoziale Charakterzüge verstärken. Soll diese Führung ihre Wirkung entfalten, muß sie präzise der individuellen Persönlichkeit angepaßt sein“; und weiter „... dürfen die Gruppen nicht zu inhomogen sein.... Das Problem des optimalen Gruppierungsschemas ist noch nicht gelöst.... Man sollte vielmehr noch individueller vorgehen, am besten nach persönlichen Affinitäten der Zöglinge zu Gruppenkameraden oder zu bestimmten Erziehern." Und zur Erzieherpersönlichkeit: „Eine ,menschliche Ordnung', wie wir sie oben forderten, verlangt ungemein viel vom Erzieher. ... Erziehung ist eine Sache des Seins und des Vorlebens; ... War eine solche bezwingende Führung gut, ... muß [man] das Kind ... nunmehr ,mit Freiheit belasten““ $[2$, 
S. 281 f.]. An dieser Stelle sei dazu auch die Lektüre des letzten Kapitels „Pädagogische Therapie" [2, S. 297-301] sine ira et studio empfohlen.

All dies sind Aussagen, die in der stationären Betreuung von „schwierigen" Kindern, egal, ob sie in modernen Wohngruppen oder Therapieheimen leben, weiterhin ihre Gültigkeit haben. Abschließend weist Asperger darauf hin, dass nicht in allen Fällen eine Heimunterbringung nötig sei, vielmehr „gute Betreuung der Familien, ... heilpädagogische Horte (oder Clubs), ... die die Kinder von ... der Straße herausnehmen, ... ihnen eine Stätte der Arbeit, des Spiels und der Bildung von Geist und Gemüt bieten," wichtig seien. Dass Asperger sehr an die Möglichkeiten ambulanter Betreuung glaubte, beweist auch die Tatsache, dass er mit seiner Arbeitsgemeinschaft für Heilpädagogik 1949 ausschlaggebend an der Gründung der ersten "child guidance clinic" in Wien beteiligt war [13, S. 52].

Ich selbst verstehe unter „strikter Führung", dass es geeignete Regeln und Strukturen braucht, um Kinder und Jugendliche mit schweren Störungen des Sozialverhaltens (so die heutige Bezeichnung) so zu "halten“ (manchmal sogar im wörtlichen Sinn), dass sie suffizient betreut und behandelt werden können. Wie wichtig dies ist, wird klar, wenn man erleben musste, einen 12-jährigen Jungen an den Straßenstrich und eine Überdosis Drogen verloren zu haben [14]. In meiner Zeit als Leiter des NÖ Heilpädagogischen Zentrums Hinterbrühl konnten wir dort, insbesondere unter der Konzeption von Rainer Fliedl (sozialtherapeutische Abteilung für Jugendliche) und Martina Grögl-Buchart (STA Kids), eine psychotherapeutische Abteilung für Kinder und Jugendliche mit diesen Problemfeldern aufbauen. Will man diesen Kindern und Jugendlichen gerecht werden, braucht ihre Betreuung eine angemessene Zahl von MitarbeiterInnen mit entsprechender Ausbildung und Persönlichkeit, ebenso wie eine bestimmte Arbeitsweise des Teams.

Betreuung und Behandlung dieser Kinder und Jugendlichen sind also personalintensiv. Nur so kann den von ihnen ausgehenden affektiven Belastungen und Spaltungstendenzen, plötzlichen Gewaltausbrüchen oder suizidalen Verzweiflungsanfällen entgegengetreten werden. Um darauf adäquat reagieren zu können, braucht es auch die Möglichkeit der Einzelbetreuung, die MitpatientInnen und BetreuerInnen, nicht zuletzt aber dem/der gewaltbereiten Kind/Jugendlichen selbst Schutz bietet. Denn werden im Laufe solcher Episoden andere Personen verletzt oder Dinge zerstört, so bedeutet dies überwiegend eine Retraumatisierung des Kindes, jedenfalls aber einen massiven Selbstwertverlust, da es sich wieder einmal als „böse“ bestätigt fühlt. Eine große Herausforderung stellt zudem die Tatsache dar, dass diese Kinder das Team massiv spalten können, indem sie in einzelnen Betreuern Gefühle wie "gut" oder „böse“ auslösen, was von diesen als persönliches Gegeneinander erlebt werden kann.

\section{Heilpädagogik insgesamt betrachtet}

Bei der Aufarbeitung der Literatur zur Bedeutung der Heilpädagogik in der Betreuung von Kindern mit besonderen Bedürfnissen lese ich v. a. folgende 2 Kritikpunkte heraus: Erstens, wie bereits ausgeführt, hätte die Heilpädagogik zu zahlreichen Missbrauchsfällen in Heimen und Wohngemeinschaften beigetragen. Zweitens sei es zur unzulässigen Machtfülle bei der Zuschreibung von Auffälligkeiten und Störungen an Kinder und Jugendliche gekommen. Dies wäre möglich geworden, durch die Monopolstellungen der Heilpädagogischen Stationen und deren vorgelagerten Diensten (Ambulanzen, Beratungsstellen und Beratungstagen an den Jugendabteilungen) und noch verschärft durch Nebentätigkeiten der Leiter/-innen als GerichtsgutachterInnen und BeraterInnen von Jugendwohlfahrtseinrichtungen [8].

Zum ersten Punkt ist, gemäß meiner persönlichen Meinung und Erfahrung nach, zu bemerken, dass nicht überall, wo „Heilpädagogik draufstand, Asperger drin war". So bezeichnete Nowak-Vogl (1922-1998) ,ihre“ Station als „heilpädagogische Beobachtungsstation des Lan- des Tirol", hatte aber keinerlei diesbezügliche Tradition aufzuweisen. Auch hatte sie nie eine Funktion in der Heilpädagogischen Gesellschaft Tirols oder gar Österreichs inne. Diese inhaltliche und ideelle Ferne von der Heilpädagogik galt auch für manche Institutionen, die sich zwar auf die „Heilpädagogik“ oder sogar auf Asperger beriefen, keineswegs aber seinen Geist internalisiert hatten.

\section{》) Nicht überall, wo „Heilpädagogik draufstand, war Asperger drin"}

Mehr noch als alle theoretischen Konzepte war aber die Ursache für die Gewalt, denen Kinder und Jugendliche in manchen Institutionen ausgesetzt waren, meiner Meinung nach dem Zusammenspiel mehrerer Faktoren geschuldet. Hierzu zählten althergebrachte Strukturen mit in sich geschlossenen Systemen, keine Begleit- oder Kontrollmechanismen, massive quantitative und qualitative Defizite bei Personal und den Leitungen sowie nicht zuletzt gesellschaftliche Vorurteile, dass die Kinder bzw. ihre Familien an ihrem Schicksal sowieso „selbst schuld seien.“ Dies findet aus meiner Sicht in den zahlreichen Studien zum Opferschutz zu wenig Beachtung.

Zum zweiten Kritikpunkt: Es ist richtig festzustellen, dass die heilpädagogischen Abteilungen/Stationen lange Jahre eine vorherrschende Rolle in der Diagnostik und der Betreuung „schwieriger" Kinder innehatten. Diese Rolle kam den heilpädagogischen Institutionen v. a. deshalb zu, weil es sonst kaum Einrichtungen gab, die diese Aufgabe auf sich nahmen. Die heilpädagogischen Abteilungen hatten also zu dieser Zeit Aufgaben zu erfüllen, die in anderen Ländern von der Kinder- und Jugendpsychiatrie abgedeckt wurden. Deren Aufgabe ist es aber nun einmal, Schwierigkeiten und Probleme mit einem Störungsnamen, also einer Diagnose zu versehen, die als Basis für die weitere Behandlung dient. Wenn man so will, ist dies Teil der „Macht“ jeder Form von Psychiatrie. Natürlich muss jedem/jeder DiagnostikerIn bewusst sein, dass das Stellen einer Diagnose auch eine Etikettierung bedeutet, weswegen er/sie 
mit der Diagnostik verantwortungsvoll umgehen muss [15].

Darüber hinaus kann ein zu enges und im Gleichklang stehendes Verhältnis zur Jugendwohlfahrt problematisch sein, da diese als Behörde Maßnahmen setzen kann und muss. Auf der anderen Seite profitieren Kinder und Jugendliche mit dissozialen Störungen durch eine gute Kooperation von einschlägigen SpezialistInnen und der Kinder- und Jugendhilfe, da sie dadurch nicht Gefahr laufen, zwischen beiden hin- und hergeschoben zu werden. Diese Kooperation muss allerdings auf Augenhöhe stattfinden, indem jeder seine Verantwortung wahrnimmt und gemeinsam mit der Familie und dem Kind bzw. dem/der Jugendlichen nach Lösungen sucht. Möglicherweise waren in der 1960er- und 1970er-Jahren das Bewusstsein und die Reflexionsbereitschaft diesbezüglich noch nicht entsprechend ausgeprägt, sodass Kinder, Jugendliche und ihre Familien gelegentlich ,unter die Räder" nichtgerechtfertigter behördlicher Maßnahmen kamen. Nach meiner Einschätzung wurde aber doch einer großen Mehrheit von KlientInnen geholfen. Aus den damals bestehenden Strukturen einen Generalverdacht gegen „die Heilpädagogik“ und die dort tätigen Personen abzuleiten, halte ich für unangemessen. Wenn in Zukunft an eine weitere Aufarbeitung gedacht wird, sollte diese in enger Zusammenarbeit von HistorikerInnen mit MedizinerInnen - auch unter Befragung von ZeitzeugInnen aus dem Betreuungspersonal und nicht nur unter Heranziehung von PatientInnenberichten - geschehen.

\section{Schlussbemerkung: „mein“ Asperger}

Bei der Lektüre der Schriften über Asperger in der Zeit des NS und danach habe ich große Schwierigkeiten damit, wie das Lebenswerk Aspergers von manchen AutorInnen anhand von Akten, Krankengeschichten und Veröffentlichungen dargestellt wird. Ich sehe darin eine Aneinanderreihung von Daten und Aussagen, die als Indizien interpretiert werden und geringe bis keine Empathie für die Person Aspergers und sein Wirken erkennen lassen. Auch finde ich es problematisch, einen Menschen vor dem Hintergrund der heutigen Ansichten und Erkenntnisse, nicht aber auch im Spiegel seiner Zeit zu sehen. So meint z. B. Friedmann [7] eine einengende Sichtweise Aspergers im Vergleich zur Zeit Lazars zu erkennen. Frankl hingegen beschreibt 1937 mit einer gewissen Begeisterung die gemeinsame prozesshafte Weiterentwicklung an der Heilpädagogischen Station: „Phänomene, die, als man sie das erste Mal sah, für einmalige Konstitutionsanomalien, eine Eigentümlichkeit des Benehmens hielt, ... gewannen jetzt, da sie bei einem zweiten und dritten Fall und immer wieder erschienen, neue Bedeutung. Man konnte genauer beobachten, sah klarer, erkannte besser kausale Verknüpfungen und gesetzmäßige Zusammenhänge. ... So entstanden und entstehen uns auch heute noch aus der Beobachtung immer mehr charakteristische Bilder der Schwererziehbarkeit" [6, S. 50].

Problematisch sehe ich auch Aussagen von Diagnosen und Befunden mit bewertenden Inhalten, die von ForscherInnen aus einem anderen Wissenschaftsgebiet abgegeben werden. So zieht Czech [5] in seinem Vergleich der Diagnosen (und Prognosen) Aspergers mit denen der Ärzte vom Spiegelgrund jene von 4 Kleinkindern heran, bei denen zwischen der Diagnosestellung Aspergers und der der anderen Ärzte ein Zeitraum von 9 bis 20 Monaten lag. Alle diese Kinder waren in dieser Zeit 4 bis 14 Monate in der Kleinkinder- und Säuglingsabteilung aufgenommen und wurden also behandelt. Eine entsprechende Publikation ist in Vorbereitung. Bei allen wird in der Krankengeschichte ein guter Behandlungserfolg beschrieben, sodass für mich als Mediziner ein Vergleich der Diagnosen vor und nach der Behandlung nicht zulässig ist. Friedmann [7] fragt, ob sich hinter der von Asperger als abnorme „Vielrednerei“ und „Vielfragerei“ beschriebenen Auffälligkeit nicht „Erkenntnisinteresse gegenüber der Umwelt" verbergen könnte. Als ob Asperger dies nicht zu unterscheiden gewusst hätte! So entstehen Bilder, die nichts Unwahres enthalten, aber doch sehr zu hinterfragen sind.

Edith Sheffer und Herwig Czech haben bewirkt, dass ich mich nach Jahrzehnten meines sehr erfüllten Berufsle- bens wieder auf die Spuren des Anfangs begeben und mich mit Hans Asperger und seinem Wirken auseinandergesetzt habe. Anfangs, indem ich intensiv den Dokumenten aus der Zeit des Nationalsozialismus nachspürte, später verwoben mit der Suche nach „meinem“ Asperger. Dabei hat das von mir errichtete „Denkmal Asperger“ ein paar Sprünge abbekommen, gleichzeitig hat der Mensch Asperger aber an „Fleisch und Blut" gewonnen.

Hans Asperger war in seiner Lehre sehr bestimmt. Als Mensch wirkte er bescheiden und freundlich; Äußerungen über sich selbst waren bei ihm immer mit einem Stück Selbstironie verbunden. Insgesamt war er eher distanziert. Dies bewirkte auch, dass es zwischen ihm und mir, als dem jüngsten Assistenten, außerhalb des eigentlichen Fachbereichs wenige persönliche Gespräche gab. Äußerungen im Zusammenhang mit der NSZeit fielen nur selten und deckten sich mit denen, die aus dem Radiointerview und Antrittsvorlesungen bekannt sind. Fragen dazu stellten wir nicht, wie wir sie auch den eigenen - fast gleichalten Eltern nicht stellten.

Bei der Auswahl der Personen, denen Asperger vertraute, hatte er nicht immer eine glückliche Hand. Seine im Prinzip sehr konservative Grundeinstellung trug sicher dazu bei, dass die Heilpädagogik (zumindest was die eigene Klinik betraf) während seines letzten Lebensjahrzehntes stagnierte.

Man merkte Asperger an, dass er gern Professor und Klinikchef war. Ob in der Vorlesung oder bei Vorträgen; er ,dozierte" gern. Er tat dies in einer geschliffenen, manchmal etwas geschraubten Sprache, wobei jeder Satz druckreif war. Dabei ging es ihm immer auch um Einstellungen und Werte, womit er viele Menschen positiv ansprach und erreichte. Es ist schwer, die Wirkung, die Asperger auf andere hatte, zu beschreiben, und es gibt nur mehr wenige, die ihn persönlich erlebt haben.

Hans Asperger wurde durch seine Erstbeschreibung einer besonderen Form des Autismus von Wing [16] und anderen auf ein Podest gestellt, das er allerdings selbst nie gesucht hat. Von diesem soll er nun, aus welchen Grün- 
den auch immer, heruntergestoßen, ja in ein schlechtes Licht gerückt werden. Es ist nicht Intention dieses Beitrags, Asperger wieder auf das Podest zu hieven. Vielmehr soll dadurch ein Beitrag geleistet werden, Aspergers Wirken und Leben aus dem Blickwinkel eines von vielen darzustellen, denen er Vorbild und Hilfe war, einen Beitrag zum Wohl der Kinder und Jugendlichen dieses Landes zu erbringen.

\section{Korrespondenzadresse}

\section{Dr. med. univ. Ernst Tatzer}

Hinterbrühl, Österreich

ernst.tatzer@kabsi.at

Dr. med. univ. Ernst Tatzer, geb. 1949, Facharzt für Kinder- und Jugendheilkunde, Facharzt für Kinderund Jugendpsychiatrie und psychosomatische Medizin. Psychotherapeut (systemische Familientherapie). Leiter des NÖ Heilpädagogischen Zentrums Hinterbrühl 1987-2011. Wissenschaftliche Arbeiten auf dem Gebiet der Kinderpsychiatrie, systemischen Therapie, Versorgung von Kindern mit besonderen Bedürfnissen und ihren Familien (NÖ Kinderplan 2016). Diverse Lehraufträge (Uni Wien, Pädagogische Akademie des Bundes in Baden, BASO/fEP St. Pölten). Ehrenpräsident der Heilpädagogischen Gesellschaft Österreich sowie der Österreichischen Gesellschaft für Kinder und Jugendpsychiatrie.

Danksagung. Mein besonderer Dank gilt Frau Prof. Dr. Maria Theresia Schubert, meiner langjährigen Kollegin und Koautorin mehrerer Arbeiten aus der Zeit an der Heilpädagogischen Station Wien, dafür dass sie mir als Reflexionspartnerin zur Verfügung stand und die Lektorierung des Artikels übernahm.

\section{Einhaltung ethischer Richtlinien}

Interessenkonflikt. E. Tatzer gibt an, dass kein Interessenkonflikt besteht. Seine persönliche Beziehung zu Hans Asperger werden im Artikel offengelegt.

Für diesen Beitrag wurden vom Autor keine Studien an Menschen oder Tieren durchgeführt. Für die aufgeführten Studien gelten die jeweils dort angegebenen ethischen Richtlinien.

\section{Literatur}

1. Asperger H (1942) "Jugendpsychiatrie" und "Heilpädagogik". Münch Med Wochenschr 89:352-356

2. Asperger H (1968) Heilpädagogik. Einführung in die Psychopathologie des Kindes für Ärzte, Lehrer, Psychologen, Richter und Fürsorgerinnen. Springer, Wien, New York

3. Asperger H (1980) Begründung und Zielsetzung heilpädagogischen Handelns. Heilpädagogik 1:80

4. Bange D (2015) Geschichte der Erforschung von sexualisierter Gewalt im deutschsprachigen Raum unter methodischer Perspektive. In: Helfferich C, Kavemann B, Kindler H (Hrsg) Forschungsmanual
Gewalt Grundlagen der empirischen Erhebung von Gewalt in Paarbeziehungen und sexualisierter Gewalt. Springer, Heidelberg, S33-49

5. Czech H (2018) Hans Asperger, National Socialism, and "race hygiene" in Nazi-era Vienna. Mol Autism 9:1-43

6. Frankl G (1937) Die Heilpädagogische Abteilung der Wiener Kinderklinik. Z Kinderschutz Fam Berufsfürsorge 29:49-54

7. Friedmann I (2016) Hans Asperger und die Heilpädagogische Abteilung der Wiener Universitätskinderklinik. Konzepte und Kontinuitäten. In Krischel M, Schmidt M, Groß D (Hrsg) Medizinische Fachgesellschaften im Nationalsozialismus Bestandaufnahme und Perspektiven. LIT, Berlin, S309-320

8. Hartig C (2017) Zusammenfassung der wesentlichen Ergebnisse. In: Dietrich-Daum E, Ralser, M, Rupnow, D (ed) Studie betreffend die Kinderbeobachtungsstation. p 49-57 der Maria Nowak-Vogl - interdisziplinäre Zugänge. https://www.uibk.ac.at/iezw/forschungen-zurkinderbeobachtungsstation/dokumente/studiekinderbeobachtungsstation-nowak-vogl-2017. pdf.Zugegriffen:7.Apr. 2020

9. Leirer I (2016) Verwaltete Kinder - Eine soziologische Analyse von Kinder- und Jugendlichenheimen im Bereich der Stadt Wien

10. Mosser P (2012) Sexuell grenzverletzende Kinder - Praxisansätze und ihre empirischen Grundlagen. Eine Expertise für das Informationszentrum Kindesmisshandlung/ Kindesvernachlässigung (IzKK). https://www. dji.de/fileadmin/user_upload/izkk/IzKK_Mosser_ Expertise.pdf.Zugegriffen:23.Apr. 2020

11. Rennert D (2018) Euthanasie und Kinderheimskandale: Aspergers böses Erbe. https:// www.derstandard.at/story/2000078268823/ euthanasie-und-kinderheimskandale-aspergersboeses-erbe.Zugegriffen: 7. Apr. 2020

12. Sheffer E (2018) Asperger's children: the origins of autism in Nazi Vienna. Norton, New York

13. Spiegl B (2012) Zu den Anfängen des Instituts für Erziehungshilfe in Wien. Diplomarbeit. Universität Wien, Wien

14. Tatzer E (2000) Festhalten oder Laufenlassen - Grenzsetzung als Mittel der Sozialisation. In: Tatzer E, Pflanzer S, Krisch K (Hrsg) Schlimm verletzt: Schwierige Kinder und Jugendliche in Theorie und Praxis. Krammer, Wien, S137-145

15. TatzerE (2003) Kinder und ihre Helfer-Werbraucht wen. In: Waldhauser $F$, Jürgenssen $O$, Püspök $R$, Tatzer E (Hrsg) Weggelegt. Kinder ohne Medizin? Czernin, Wien, S111-139

16. Wing $L$ (1981) Asperger's Syndrome: a clinical account. Psychol Med 11:115-129 